\title{
Ein Bolusrechner hilft, die Therapieziele zu erreichen
}

\author{
Die Diabetestherapie sollte bei guter Blutzuckereinstellung mög- \\ lichst individuell und flexibel sein. Diabetiker, die ihre Basis-Bolus- \\ Insulintherapie an aktuelle Gegebenheiten anpassen wollen, \\ brauchen dafür einiges an Rechenleistung. In einer aktuellen Stu- \\ die wurde nun bestätigt, dass ein ins Blutzuckermessgerät inte- \\ grierter Bolusrechner dabei eine effektive Hilfe sein kann.
}

Die manuelle Bolusberechnung zur Anpassung der kurz wirksamen Insulindosis an Mahlzeiten oder Aktivitäten ist für viele Patienten schwierig und hat eine Menge Fehlerquellen. Oft gelingt es zum Beispiel nicht, das noch im Körper zirkulierende Insulin korrekt einzuschätzen, was das Risiko für Hypoglykämien erhöht. Bei der Insulinpumpentherapie haben sich in die Pumpe integrierte Bolusrechner bereits etabliert. Um zu prüfen, wie effektiv, sicher und akzeptiert ein solcher Rechner bei Patienten mit manueller Basis-Bolus-Insulintherapie ist, wurde die Studie ABACUS (Automated Bolus Adviser Control and Utility Study) initiiert.

Wie Dr. Ian Cranston vom Queen Alexandra Hospital in Portsmouth, UK, berichtet hat, wurden dafür 218 unzureichend eingestellte Diabetiker randomisiert, 202 vom Typ 1, die übrigen vom Typ 2, von denen 193 die 26-wöchige prospektive deutsch-britische Studie beendeten. Der Ausgangs-HbA $\mathrm{Hc}_{1 \mathrm{c}}$-Wert der Patienten lag bei im Mittel etwa 8,9\% und die Insulintherapie bestand seit mehr als 10 Jahren. Primäres Ziel der Studie war es, den $\mathrm{HbA}_{1 \mathrm{c}}$-Wert um mehr als 0,5\%-Punkte zu senken. Zusätzlich wurden unter anderem die Adhärenz der $\mathrm{Pa}$ tienten, die Glukosevariablität, die Häufigkeit von Hypoglykämien und die Therapiezufriedenheit erfasst.

Die Interventionsgruppe erhielt den AkkuChek $^{\circledast}$ Aviva Expert, ein Blutzuckermessgerät mit integriertem Bolusrechner, der patientenspezifische Daten in die Berechnung der Insulinkorrektur einbezieht. Die Kontrollgruppe erhielt zur Blutzuckermessung den AkkuChek Aviva Nano ohne Bolusrechner und musste Korrekturboli selbst berechnen. Alle Patienten wurden entsprechend geschult, regelmäßig einbestellt und erstellten zu Beginn, zur Halbzeit und am Ende

\section{Rote Liste 2013 erschienen}

Die ROTE LISTE ${ }^{\oplus}$ bietet:

- Orientierung an Indikationen tel

Internet: www.rote-liste.de der Studie strukturiert 7-Punkt-Blutzuckermessprofile. Bei etwa der Hälfte der Patienten erfolgte verblindet zusätzlich ein kontinuierliches Glukosemonitoring.

\section{Profitieren besonders jüngere \\ Diabetiker vom Bolusrechner?}

Erste noch unpublizierte Ergebnisse der Studie, die Cranston in Paris präsentierte, waren wie folgt: Insgesamt fiel der $\mathrm{HbA}_{1 \mathrm{c}}$-Wert im Mittel um 1,2\%-Punkte ab. „Allerdings erreichten die Patienten in der Interventionsgruppe mit 56\% versus $34,4 \%$ fast doppelt so häufig eine $\mathrm{Re}$ duktion um mehr als 0,5\%-Punkte als die Kontrollgruppe.“ Der Unterschied war signifikant. Aufgeteilt in die Altersgruppe 18-30 Jahre und älter als 30 Jahre profitierten beide Altersklassen, allerdings erreichten bei den Jüngeren dieje-

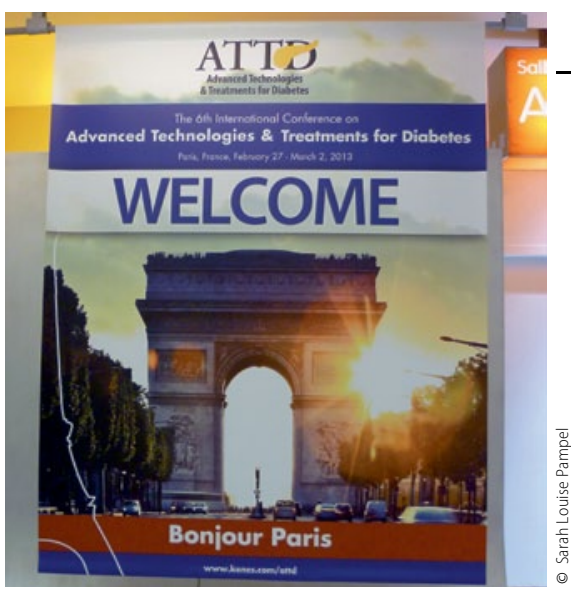

nigen öfter das gesteckte Therapieziel, die einen Bolusrechner hatten. Und dies, obwohl sie seltener seinen Rat suchten und befolgten als die Älteren. Möglicherweise profitierten gerade schwerer einstellbare jüngere Patienten von dieser Technologie, sagte Cranston. Insgesamt suchten drei Viertel der Patienten den Rat des Bolusrechners und befolgten ihn dann in $90 \%$ der Fälle.

Eine signifikante Abnahme der Glukosevariabilität wurde nur bei den Über-30Jährigen mit Bolusrechner verzeichnet. Die Patienten der Interventionsgruppe gaben zudem signifikant häufiger eine verbesserte Therapiezufriedenheit an. Das Erreichen des primären Endpunkts war nicht mit einer erhöhten Häufigkeit schwerer Hypoglykämien assoziiert.

Sarah Louise Pampel

Quelle: Roche Diabetes Care Media Talk am 27.2.2013, ATTD-Kongress in Paris, Veranstalter: Roche Diabetes Care

Die ROTE LISTE ${ }^{\circledR} 2013$ ist seit April verfügbar. Die aktualisierte Buchausgabe des Arzneimittelverzeichnisses umfasst rund 24.000 Medikamente. Diese sind in 6482 Präparateeinträgen mit 7887 Darreichungsformen und 23848 Preisangaben von 457 pharmazeutischen Unternehmen sowie von Vertreibern/Herstellern bestimmter Medizinprodukte zusammengefasst.

Nach einer Umfrage 2012 bei den Nutzern des Buchs wollen rund 90\% der Teilnehmer nicht darauf verzichten.* Für sie steht die ROTE LISTE ${ }^{\circledast}$ für verlässliche, profunde Informationen rund um Arzneimittel und ausgewählte Medizinprodukte. Dreiviertel der Teilnehmer nutzen die Buchausgabe mindestens $1 \mathrm{mal}$ wöchentlich, auch als prägnante Zusammenfassung der Fachinformation.

- Prägnante, kurz gefasste Produktinformationen

- Zusammenfassende Signaturen für die Übersicht von Gegenanzeigen, Anwendungsbeschränkungen, Nebenwirkungen, Wechselwirkungen, Intoxikationen, Warnhinweisen und Hinweisen von Wirkstoffgruppen

- Hintergrundinformationen für die Praxis in den Sonderkapi-

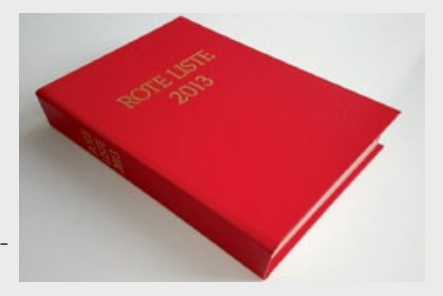

Die ROTE LISTE ${ }^{\circledR}$ richtet sich an Anwender (Heilberufe), Entscheider im

Gesundheitswesen sowie pharmazeutische Unternehmer.

Die ROTE LISTE 2013 ISBN-13: 978-3-939192-70-1,

Abonnement: 978-3-939192-71-8, Preis: 78.- € inkl. MwSt., zzgl. Versandkosten,

${ }^{*}$ Mit dem Buchversand der ROTE LISTE 2012 durchgeführte Umfrage unter den Nutzern.

Quelle: Rote Liste ${ }^{\bullet}$ Service $\mathrm{GmbH}$ 